\title{
Application of Broccoli Residues to Soil can Suppress Verticillium Wilt of Cotton by Regulating the Bacterial Community Structure of the Rhizosphere
}

\section{Weisong Zhao}

Institute of plant protection,hebei academy of agricultural and forestry sciences

\section{Qinggang Guo}

Institute of plant protection, hebei academy of agricultural and forestry sciences

\section{Shezeng Li}

Institute of plant protection,hebei academy of agricultural and forestry sciences

Xiuyun Lu

Institute of plant protection, hebei academy of agricultural and forestry sciences

\section{Peipei Wang}

Institute of plant protection,hebei academy of agricultural and forestry sciences

Lihong Dong

Institute of plant protection, hebei academy of agricultural and forestry sciences

\section{Xiaoyun Zhang}

Institute of plant protection,hebei academy of agricultural and forestry sciences

\section{Zhenhe Su}

Institute of plant protection,hebei academy of agricultural and forestry sciences

\section{Ping Ma ( $\sim$ pingma88@126.com )}

Institute of plant protection,hebei academy of agricultural and forestry sciences

\section{Research Article}

Keywords: verticillium wilt, broccoli residues, rhizosphere bacterial community structure, Bacillus subtilis

Posted Date: July 9th, 2021

DOl: https://doi.org/10.21203/rs.3.rs-607945/v1

License: (c) (1) This work is licensed under a Creative Commons Attribution 4.0 International License.

Read Full License 


\section{Abstract}

[Aims] Verticillium wilt (VW) of cotton was effectively controlled by application of broccoli residues (BR) to soil. Information regarding the variation in bacterial communities in rhizosphere of cotton cultivars with different VW resistance levels under BR treatment is still lacking and finally to provide guidance for screening effective biocontrol bacteria.

[Methods] Real-time fluorescence quantitative PCR was used to determine the population of Verticillium dahliae, the effects of BR on the bacterial community structure in rhizosphere were determined by highthroughput sequencing technology.

[Results] Results showed that control effects for susceptible cultivar (cv. EJ-1) and resistant cultivar (cv. J863) on VW after BR treatment were $51.76 \%$ and $86.15 \%$, the population of $V$. dahliae decreased by $18.88 \%$ and $30.27 \%$, respectively. High-throughput sequencing showed that ACE and Chao 1 indices were increased by application of BR. Actinobacteria, Proteobacteria, Bacteroidetes, Gemmatimonadetes, Acidobacteria, and Firmicutes were the most dominant phyla, and relative abundances of these bacterial taxa significantly differed between cultivars. Additionally, Bacillus stably increased in rhizosphere following BR treatment. Redundancy analysis (RDA) showed that relative abundances of Bacillus, Lysobacter, Streptomyces, Rubrobacter, Gemmatimonas, Bryobacter and Nocardioides were correlated with occurrence of VW. Field experiments demonstrated that dressing cotton seeds with Bacillus subtilis NCD-2 could successfully reduce occurrence of VW, and control effects for EJ-1 and J863 were $35.26 \%$ and $31.02 \%$, respectively.

[Conclusions] The application of BR changed the bacterial community structure in cotton rhizosphere, decreased the population of $V$. dahliae in soil, and increased the abundance of beneficial microorganisms, thus significantly reducing the occurrence of VW.

\section{Introduction}

Cotton (Gossypium hirsutum L.) is the most important source of natural textile fibers worldwide and a significant oilseed crop (Zhang et al., 2016). Verticillium wilt (VW), caused by Verticillium dahliae, is a typical soil-borne disease and results in extensive economic losses. In China, losses of approximately 250-310 million US dollars have been reported for cotton annually due to V. dahliae (Li et al., 2015; Rehman et al., 2018). VW is particularly difficult to control due to the long-living dormant microsclerotia produced by the pathogen, which remain viable in the soil for more than two decades (Fradin and Thomma, 2006; Alstrom, 2001), as well as the inability of fungicides to contact the hyphae of $V$. dahlia after they spread inside the xylem (Klosterman et al. 2009). It is imperative to develop novel control strategies to control this devastating disease.

Previous studies have shown that soil-borne disease management has relied principally upon fumigation (Atallah et al. 2012; Johnson and Dung, 2010; Taylor et al., 2005). However, the application of chemical fumigants to the soil may be environmentally unfriendly (Uppal et al., 2008). Therefore, there is growing 
interest in the search for alternatives to fumigants for disease control. Many reports have demonstrated that the use of organic soil amendments may be a potential strategy for the control of insect pests, pathogens, nematodes, and weeds (Díaz-Pérez, 2007; López-Pérez et al., 2005; Koike and Subbarao 2000); they can increase organic matter and nutrients and change the structure of the microbial community in the soil (Kavino et al., 2010; Shen et al., 2013; Akao et al., 2017). The changes in soil microbial community structure caused by organic soil amendments provide useful information on soil health and quality (Poulsen et al., 2013). In particular, the responses of soil bacterial communities to organic soil amendments are particularly important and are believed to be one of the main drivers of disease suppression (Garbeva et al., 2004). The disease-suppressive effects of certain crop residues are well documented, such as those of broccoli, buckwheat, canola, mustard, and sweet corn (Tubeileh and Stephenson 2020; Housam et al., 2018; Antoniou et al., 2017; Manuel and Celia 2017; Romanyà et al., 2019; Zhao et al., 2019a). Broccoli residues were reported to successfully decrease the incidence of VW in eggplant, potato, strawberry, sunflower and cotton by reducing microsclerotia or DNA copies of $V$. dahliae in the soil (Inderbitzin et al., 2018; Zhao et al., 2019a; Zhao et al., 2021). Broccoli residues also suppress weeds, nematodes, and pests (Koike and Subbarao 2000; Guerra et al., 2017).

As described in our previous work, VW of cotton was effectively controlled by the application of broccoli residues in greenhouse and field experiments (Zhao et al., 2019a; Wang et al., 2020). Recent evidence suggests that variability in plant genotypes or cultivars can have a significant impact on rhizosphere microbiomes, particularly bacteria (Stringlis et al., 2018; Zhang et al., 2019). Information regarding the variation in bacterial communities in rhizosphere soil that are affected by cotton cultivars that vary in resistance to VW following the application of broccoli residues is still lacking. It is unclear whether and, if so, how cultivar resistance against $V$. dahliae is related to rhizosphere bacteria.

The overall objectives of this study were therefore (i) to determine the effect of BR on the incidence of VW among different cotton cultivars, (ii) to study the differences in the bacterial composition, diversity, and community structure following the application of BR, (iii) to analyze the relationship between disease incidence and the bacterial community, and (iv) to assess the effect of the addition of exogenous Bacillus subtilis on VW in the field.

\section{Materials And Methods}

\section{Field experiment site}

The experimental sites were located in Quzhou County, Hebei Province. Field trials were conducted at two sites, Field A and Field B, from 2017 to 2019. The experimental sites have a long history of cotton cultivation and occurrence of VW. The plots with flat terrain, relatively uniform fertility and continuous cotton planting for more than ten years were selected as experimental fields. Soil nutrient characteristics are outlined in detail in a previous publication (Zhao et al., 2019b). Detailed information regarding the field experiment setting is described in the following statements.

\section{Experimental setup and design}


Broccoli was planted in August 2017, and the density of the broccoli plants was approximately 41 thousand plants per hectare. After harvesting the edible part of the broccoli, the remaining parts of the plants were chopped in the field with a grinder and mechanically incorporated into the soil with a rotovator in early November 2017 at a depth of 25 to $30 \mathrm{~cm}$. The amount of broccoli residues amended into the soil was approximately 57 thousand kilograms per hectare. Parts of the field were not amended with broccoli residues as a blank control. The susceptible cultivar Ejing 1 (EJ-1) and resistant cultivar Ji 863 (J863) were planted in late April 2018. The experimental design included four treatments: 1) susceptible cultivar EJ-1 planted without broccoli residues (EJ-1-CK); 2) susceptible cultivar EJ-1 planted with broccoli residues (EJ-1-BR); 3) resistant cultivar J863 planted with broccoli residues (J863-BR); 4) resistant cultivar J863 planted without broccoli residues (J863-CK). The experiment had a randomized complete block design with three replicates. All plots were covered with plastic film and irrigated as necessary. For Field B, in early August 2018, broccoli planting, preparation of BR, and experimental setup and design were similar to those in Field A. After that, cotton was planted in late April 2019.

\section{Soil sample collection and DNA extraction}

Soil samples were collected at the flowering and boll-forming stages in 2018 and 2019, respectively. Within each sampling plot, three plants were randomly selected and carefully removed from the soil using a spade. The root systems of the three plants from each plot were first vigorously shaken to remove loosely adhering soil particles, and then the remaining root systems were combined as a rhizosphere sample. Soil samples were immediately preserved at $4^{\circ} \mathrm{C}$ for less than $48 \mathrm{hr}$. To remove plant material, the sample was sieved through a $2.0 \mathrm{~mm}$ sieve and stored at $-80^{\circ} \mathrm{C}$ for subsequent DNA extraction. DNA was extracted following the instruction manual for the FastDNA ${ }^{\mathrm{TM}}$ SPIN Kit for Soil (MP Biomedicals, Solon, $\mathrm{OH}$, USA) in accordance with the protocol of the manufacturer. The concentration and quality of the DNA were determined using a spectrophotometer (NanoDrop 2000, Thermo Fisher Scientific Inc., Waltham, MA, USA). The extracted DNA was stored at $-20^{\circ} \mathrm{C}$ prior to further analyses.

PCR amplification, Illumina MiSeq sequencing and DNA copies of Verticillium dahliae

PCR amplification of bacterial 16S rRNA targeting the V3/V4 region was conducted by using primers 338F (5'-ACTCCTACGGGAGGCAGCA-3') and 806R (5'-GGACTACHVGGGTWTCTAAT-3'). PCR protocols were used to amplify the 16S rRNA gene (Lu et al., 2018). Finally, equal amounts of PCR product from each sample were placed in individual tubes and analyzed with the Illumina MiSeq platform. Illumina MiSeq sequencing was performed at Majorbio Biopharm Technology Co., Ltd. (Shanghai, China). The DNA copies of $V$. dahliae in the different soils were studied with real-time fluorescence quantitative PCR according to the method described in our previous study (Zhao et al, 2019a).

\section{Verification of the biocontrol function of Bacillus subtilis NCD-2 against VW in the field}

To examine the effect of the addition of exogenous $B$. subtilis NCD-2 on cotton VW, two treatments were applied for each cultivar as described below. (1) cotton seeds were coated with $B$. subtilis $(10 \%, \mathrm{w} / \mathrm{w})$ at a spore concentration of $10^{9} \mathrm{CFU} / \mathrm{g}$ (representing BS), and (2) cotton seeds were not treated with B. subtilis 
(representing CK). The experiment had a randomized complete block design with three replicates in the field. The experiment was carried out in 2019.

\section{Disease Evaluation}

VW severity on all individual plants was recorded on a scale of 0 to 4 . The disease index for each plot was calculated based on a five-level categorization of cotton VW according to the percentage of plant leaves with symptoms such as chlorosis, necrosis or defoliation. $0=$ healthy plants or no symptoms, $1=$ diseased plants with leaf symptoms below $25 \%, 2=26-50 \%$ diseased plants with leaf symptoms and leaf margin rolled up and showing symptoms of scorch, $3=51-75 \%$ diseased plants with leaf symptoms and leaf margin rolled up with wilting symptoms, $4=$ more than $76 \%$ diseased plants or dead with leaf symptoms. The disease incidence, disease index, and control effect were calculated using the following formula:

Disease incidence $(\%)=($ No. of diseased plants $/ 30) \times 100$

Disease index $=\left[100 \times \sum\right.$ (No. of diseased plants $\times$ responding disease rating) $] /(30 \times 4)$

Control effect $(\%)=[$ (disease index of CK - disease index of treatment)/disease index of CK $] \times 100$

\section{Statistical Analyses}

Statistically significant differences $(P<0.05)$ in disease incidence, the disease index, DNA copies of $V$. dahliae, and changes in the bacterial community composition between the control and BR treatments were evaluated with Student's t-test or one-way analysis of variance (ANOVA) using SPSS. Soil bacterial diversity indices were calculated based on resampled OTU abundance matrices in MOTHUR. Principal component analysis (PCA) was performed to explore the differences in soil bacterial community composition. Redundancy analysis (RDA) was performed to examine the relationship between disease occurrence and bacterial community composition. Analysis of similarities (ANOSIM) was performed to identify the significant differences in bacterial community structure among treatments. Data on the differences in bacterial community composition among treatments were obtained, and the relative abundances of major taxonomic groups at the phylum and genus levels were compared. Graphs were generated with Origin 8.0 software.

\section{Results}

\section{Effects of broccoli residues on VW of different cotton cultivars}


Broccoli residues had a significant impact on the disease incidence and disease index of cotton VW (Pष0.05). Compared with the blank control (no broccoli residues), the disease incidence of cultivar EJ-1 decreased by $38.76 \%$ and $53.50 \%$ and the disease index decreased by $46.47 \%$ and $57.04 \%$ in Field $A$ and Field B, respectively. The disease incidence of cultivar $J 863$ decreased by $100 \%$ and $63.42 \%$ and the disease index decreased by $100 \%$ and $72.30 \%$ in Field $A$ and Field B, respectively. The average control effects for EJ-1 and J863 were $51.76 \%$ and $86.15 \%$, respectively (Fig. 1).

\section{Effect of broccoli residues on DNA copies of V. dahliae in soil}

When compared with those in the blank control soils, the DNA copies of $V$. dahliae in the soils associated with the different cotton cultivars were significantly reduced by the BR treatment (Fig. 2). For EJ-1, the DNA copies of $V$. dahliae decreased by $14.31 \%$ and $23.44 \%$ in Field A and Field B, respectively. For J863, the DNA copies of $V$. dahliae decreased by $34.19 \%$ and $26.34 \%$, respectively. The average numbers of DNA copies of $V$. dahliae for EJ-1 and J863 were decreased by $18.88 \%$ and $30.27 \%$ following the application of BR, respectively.

\section{Alpha Diversity Of The Bacterial Community}

The alpha diversity of the bacterial community was expressed by the ACE and Chao1 indices in our study (Fig. 3). In Field A, the ACE index for EJ-1 ranged from 2503 (CK) to 2667 (BR), and the Chao1 index ranged from 2501 (CK) to 2624 (BR), which were greater by $6.55 \%$ and $4.92 \%$, respectively. The ACE index for J863 ranged from 2603 (CK) to 2652 (BR), and the Chao1 index ranged from 2585 (CK) to 2617 (BR), which were greater by $1.89 \%$ and $1.24 \%$, respectively. In Field $B$, the ACE index for EJ-1 ranged from 3690 (CK) to 3751 (BR), and the Chao1 index ranged from 3466 (CK) to 3541 (BR), which were greater by $1.64 \%$ and $2.15 \%$, respectively. The ACE index for J863 ranged from 3949 (CK) to 3972 (BR), and the Chao1 index ranged from 3520 (CK) to 3655 (BR), which were greater by $0.59 \%$ and $3.85 \%$, respectively. These results indicate that the ACE and Chao1 indices were increased by the application of BR at the different field sites.

\section{Bacterial Community Structure Analyses}

Principal component analysis based on the OTU composition was used to study the effect of broccoli residues on the soil bacterial community structure associated with the different cotton cultivars. Figure 4 shows plots of the sites in the plane of the first two principal coordinates based on the soil bacterial communities in Field A and Field B, respectively. The results show that the bacterial community structure associated with the different cultivars was located in the same quadrant after the application of broccoli residues, while that of the blank controls of the different cultivars was located in different quadrants, which indicates that the bacterial community structure changed and tended to be the same after the application of broccoli residues. In addition, the first principal component (PC1) and the second principal component (PC2) of the bacterial community structure at the OTU level in rhizosphere soil were found to 
explain $34.07 \%$ and $16.36 \%$ of all variables in Field A, and $24.83 \%$ and $21.29 \%$ of all variables in Field B, respectively. The cumulative contribution rates of variance of the two principal components reached $50.43 \%$ and $46.12 \%$, respectively. In addition, ANOSIM indicated that the BR treatment contributed significantly to the separation of the $C K$ treatment $(R=0.9815, P=0.001$, Field $A)$ and $(R=0.6481, P=$ 0.002 , Field B).

\section{Comparison Of Bacterial Community Composition}

Among all sequences, unknown sequences were classified as "other group". In Field A, the dominant bacterial phyla were Proteobacteria, Actinobacteria, Acidobacteria, Gemmatimonadetes, Chloroflexi, Bacteroidetes, Planctomycetes, Rokubacteria, Nitrospirae, Verrucomicrobia, Latescibacteria, Firmicutes and Patescibacteria, and these phyla accounted for more than $95 \%$ of the total sequences in each sample (Fig. S1). The changes in the relative abundances of the dominant bacterial taxa associated with the different cotton varieties after the application of broccoli residues were compared at the phylum level (Fig. 5). Notably, all dominant bacterial phyla associated with J863 increased in abundance following the application of broccoli residues, while for EJ-1, the dominant bacterial phyla were influenced to different degrees by the application of the broccoli residues. Among them, Gemmatimonadetes, Rokubacteria, Nitrospirae, Verrucomicrobia and Firmicutes increased. The most abundant group was Firmicutes, which increased by approximately 2.36 and 1.41 -fold for EJ-1 and J863, respectively, when compared with the values for CK. However, Proteobacteria, Chloroflexi, Bacteroidetes, Planctomycetes, Latescibacteria and Patescibacteria decreased following the application of broccoli residues (Fig. 5A). In Field B, the dominant bacterial phyla were Actinobacteria, Proteobacteria, Acidobacteria, Chloroflexi, Gemmatimonadetes, Firmicutes, Bacteroidetes, Planctomycetes, Rokubacteria, Patescibacteria, Entotheonellaeota, Nitrospirae and Verrucomicrobia, and these phyla accounted for more than $95 \%$ of the total sequences in each sample (Fig. S2). For the cultivars EJ-1 and J863, the dominant bacterial phyla were influenced to different degrees by treatment with broccoli residues. Actinobacteria, Gemmatimonadetes and Firmicutes were increased by the application of broccoli residues. The most abundant group was also Firmicutes in Field B, and the fold changes were 1.42 and 1.27 for EJ-1 and J863, respectively. Acidobacteria decreased as a result of the application of broccoli residues. In addition, Proteobacteria, Bacteroidetes, Patescibacteria, Entotheonellaeota, Nitrospirae and Verrucomicrobia decreased for EJ-1, while the opposite tendency was observed for J863 (Fig. 5B). Based on the results for the different cultivars and field sites, the relative abundances of Actinobacteria, Gemmatimonadetes and Firmicutes in the soil increased after the application of broccoli residues.

In Field A, the dominant bacterial genera (cultured and at least one group in two groups average relative abundance >0.5\%) were Sphingomonas, RB41, MND1, Haliangium, Nitrospira, Lysobacter, Bryobacter, Gemmatimonas, Gaiella, lamia, Pontibacter, Streptomyces, Steroidobacter, Ilumatobacter, Blastococcus, Dongia, Luedemannella, Rubrobacter, Nocardioides, Gemmatirosa, Massilia, Bacillus and Sphingobacterium (Fig. 6A). Among them, the relative abundances of RB41, Gemmatimonas, Pontibacter, Streptomyces, Blastococcus, Massilia and Bacillus were increased by the application of broccoli residues 
for all cultivars, while decreases in MND1, Nitrospira, Bryobacter, lamia, Steroidobacter, Ilumatobacter and Dongia were observed after the application of broccoli residues.

In Field B, the dominant bacterial genera were Arthrobacter, Streptomyces, RB41, Rubrobacter, Bacillus, Sphingomonas, Nocardioides, Solirubrobacter, MND1, Gaiella, Blastococcus, Steroidobacter, Bryobacter, Nitrospira, Marmoricola, Microvirga, Microlunatus, Haliangium, Skermanella, Dongia, Intrasporangium, Lysobacter, Gemmatimonas, Novosphingobium, Kribbella and Nitrolancea (Fig. 6B). Among them, the relative abundances of Arthrobacter, Bacillus, Microvirga, Intrasporangium, Lysobacter and Nitrolancea increased, while those of Streptomyces, MND1, Blastococcus, Steroidobacter, Nitrospira, Haliangium, Dongia and Kribbella decreased. Based on the above results at the two field sites, Bacillus was the only genus that steadily increased after the application of broccoli residues.

\section{Relationships between the occurrence of $\mathrm{VW}$ and bacterial community composition}

The relationships between the occurrence of VW and bacterial community composition in Field $A$ and Field B were studied with RDA (Fig. 7). For Field A, the RDA that was performed with the genera and disease incidence data showed that the first two RDA components could explain $52.3 \%$ of the total variation (Fig. 7A). As shown by their close grouping and by the vectors, the disease incidence of cultivar J863 was positively related to the abundant genera Gemmatimonas, Pontibacter, RB41, Blastococcus and Massilia after the application of broccoli residues, and it was negatively related to Bacillus, Lysobacter, and Nitrospira. However, the disease incidence for EJ-1 treated with BR was positively related to the abundant genera Streptomyces, Rubrobacter, Bryobacter and Nocardioides, and it was negatively related to Gemmatimonas, Pontibacter, RB41, Blastococcus and Massilia.

For Field $\mathrm{B}$, the RDA that was performed with the genera and disease incidence data showed that the first two RDA components could explain $47 \%$ of the total variation (Fig. 7B). The disease incidence in the BR treatment for the cultivars (including J863 and EJ-1) was positively related to the abundant genera Bacillus, Nocardioides, RB41, Rubrobacter, and Arthrobacter and negatively related to Streptomyces, Nitrospira, Sphingomonas, and Lysobacter.

\section{Control effect of the exogenous application of Bacillus subtilis NCD-2 on VW}

The control effect of the application of $B$. subtilis on cotton VW was investigated in our study. As indicated in Fig. 8, the control effect of BS on the disease at the boll-forming stage for EJ-1 was 38.55\%, while that for J863 was $26.73 \%$. Additionally, further study at boll opening showed that the control effect for EJ-1 was $31.96 \%$, while that for J863 was $35.31 \%$. The average control effects for EJ-1 and J863 were $35.26 \%$ and $31.02 \%$, respectively.

\section{Discussion}

The use of crop residues is an important method associated with the suppression of VW, such as those of broccoli, buckwheat, canola, mustard, and sweet corn (Subbarao et al., 1999; Wiggins and Kinkel, 2005; 
Inderbitzin et al., 2018; Wheeler and Johnson, 2016; Zhao et al., 2019a; Zhao et al., 2021). In this study, resistant and susceptible cultivars of cotton VW were chosen to evaluate the effect of the application of broccoli residues on the occurrence of VW. The results further confirmed that treatment with BR could effectively reduce the incidence of VW among different cotton cultivars and decrease the population of $V$. dahliae in soil. These results are consistent with those from previous studies on disease in cauliflower, eggplant, and potato after treatment with broccoli residues (Inderbitzin et al., 2018; Ikeda et al., 2015; Davis et al., 2010; Subbarao et al., 1999). Therefore, the application of broccoli residues provides a new method and ideas for the sustainable ecological control of cotton VW. In our previous study, the main potential mechanism by which broccoli residues incorporation into the culture substrate reduced the DNA copies of $V$. dahliae and inhibited the spread of $V$. dahliae was revealed by real-time PCR and confocal microscopy methods (Wang et al., 2020; Zhao et al., 2019a). However, more in-depth research should be performed to further explore this potential mechanism, especially from the perspective of rhizosphere microbiomics. Microbiome-based research has opened a new frontier that will greatly expand our knowledge of the relationship between plant disease incidence and microbiota and offer new opportunities for developing novel approaches for biocontrol. To our knowledge, although the Illumina MiSeq high-throughput technique has been used to study the effects of cultivation or fallowing, crop rotation, and the application of common composts on the soil bacteria community composition or structure (Tian et al., 2017; Chamberlain et al., 2020; Gong et al., 2021), this detailed comparison of the soil bacteria community associated with different cotton cultivars resistant to VW after the application of broccoli residues was the first to be performed with the Illumina MiSeq method.

There are differences in opinion on the relationships between bacterial community indices (richness and diversity) and disease incidence (Bulluck et al., 2002; Shen et al., 2014). Many studies have shown that the diversity and composition of the soil microbial community are related to the occurrence of soil-borne diseases (Gamliel et al., 2000; Bulluck et al., 2002; Zhao et al., 2021). However, Shen et al. (2014) found that there was no significant correlation between bacterial community indices and banana Fusarium wilt following the application of bioorganic fertilizer. In the present study, although there were no significant differences in the alpha diversity indices following the application of BR, the values of those indices increased. Moreover, the treatment with broccoli residues had a significant impact on the soil bacterial community structure, which was consistent with the results of previous studies on the changes in bacterial community structure in the rhizospheric soil of eggplant (Inderbitzin et al., 2018). In addition, the bacterial community structure associated with the different resistant cotton cultivars changed in comparison to that in CK and was located in the same quadrant after the application of broccoli residues, indicating that the bacterial community structure tended to be the same after the application of broccoli residues (Fig. 4). In terms of the bacterial community composition, the analysis at the phylum level revealed that Actinobacteria, Proteobacteria, Bacteroidetes, Gemmatimonadetes, Acidobacteria, and Firmicutes were the most common phyla, but with some changes in relative abundance. This finding roughly corresponded with those of previous articles (Inderbitzin et al., 2018; Shen et al., 2014). Inderbitzin et al. (2018) found that the five dominant phyla of soil bacteria were Proteobacteria, Actinobacteria, Bacteroidetes, Firmicutes, and Acidobacteria. Among them, Actinobacteria and 
Proteobacteria were more abundant after treatment with broccoli residues than in the control, while the opposite tendency of Bacteroidetes, Firmicutes, and Acidobacteria was observed. Our study found that the relative abundances of Actinobacteria and Proteobacteria also increased for the resistant cultivar J863, which was consistent with previous studies (Inderbitzin et al., 2018). However, there was no consistent conclusion for these two phyla for the susceptible cultivar EJ-1. The reason for this finding is still unclear and may be due to differences in the types or contents of root exudates among cotton cultivars, which can cause different microbial communities to be recruited. Moreover, the relative abundance of Firmicutes in the soil associated with different resistant cotton cultivars increased after the application of broccoli residues, which was not consistent with the findings of Inderbitzin et al. (2018).

Analysis of the dominant genera also revealed significant differences in the bacterial communities among the different treatments. Among all the cotton cultivars, the abundance of Bacillus was increased by treatment with the broccoli residues. RDA showed that the incidence of VW might be positively related to MND1, Steroidobacter, Nitrospira and Dongia, which belong to Proteobacteria and Nitrospirae. Moreover, we found that the Gemmatimonas (Gemmatimonadetes phylum) and Streptomyces genera (Actinobacteria phylum) might respond to the suppression of $\mathrm{VW}$ by treatment with broccoli residues in Field A and that Lysobacter (Proteobacteria phylum) might do so in Field B. Yin et al. (2013) reported that members of the Gemmatimonas genus were found at a relatively high frequency in the rhizosphere of healthy plants. Many articles have reported that Streptomyces and Lysobacter could be used as biological control agents against plant pathogens (Hao et al., 2019; Puopolo et al., 2015). Bacillus, which belongs to Firmicutes, plays an important role in the biological control of plant disease (Wang et al., 2020; Roy et al., 2018; Pandin et al., 2018). Previous studies have reported the potential of Bacillus sp. in the biological control of VW of cotton, potato, eggplant, sunflower, and olive (Azabou et al., 2019; Zhao et al., 2020). Bacillus subtilis NCD-2 was first isolated from cotton rhizosphere soil in Hebei Province and showed excellent biological control of soil-borne diseases (Li et al., 2005). In the present study, Bacillus significantly increased in abundance after the application of broccoli residues. Therefore, to verify the control effect of $B$. subtilis NCD-2 against VW, field experiments were executed by seed dressing with $B$. subtilis. However, the control effect of strain NCD-2 on disease was approximately $35 \%$ for different cotton cultivars. Some researchers have reported that direct applications of potentially beneficial species often result in poor disease suppression due to their low survival and colonization in soil (Saravanan et al., 2003; Lugtenberg and Kamilova, 2009). Therefore, the survival or abundance of the biocontrol inoculant $B$. subtilis in rhizosphere soil will be studied in future research.

In addition, many studies have shown that soil physical and chemical properties such as soil nutrients, $\mathrm{pH}$ and organic matter are important factors affecting the structure of the soil microbial community (Zhao et al., 2020; Cui et al., 2017; Zhou et al., 2015). Furthermore, soil temperature and humidity are also important environmental factors affecting soil microbial community structure (Bastida et al., 2018; Zhou et al., 2017) and need to be studied in the future.

\section{Conclusions}


In this study, the incidence of cotton VW and the population of $V$. dahliae in the rhizosphere of cotton cultivars with different verticillium wilt resistance levels were decreased by treatment with BR. Highthroughput sequencing showed that bacterial diversity was increased by the application of BR. The relative abundances of Bacillus, Lysobacter, Streptomyces, Rubrobacter, Gemmatimonas, Bryobacter and Nocardioides were correlated with the occurrence of verticillium wilt. These results provide important information necessary for a better understanding of bacterial community structure in rhizosphere soil after treatment with BR.

\section{Declarations}

\section{Data availability statement}

The raw sequence data reported in this paper have been deposited at the National Center for Biotechnology Information (NCBI) under accession number PRJNA734729 and PRJNA734770.

\section{Author contributions}

WZ, QG, SL, and PM planned, designed the research, and experiments. WZ, PW, LD, XZ, ZS, and XL performed the experiments. WZ and PM analyzed the data and wrote the manuscript. All authors read and approved the final manuscript.

\section{Acknowledgments}

This work was supported by the National Key Research and Development Program of China (2017YFD0200601) and Special Fund for Agro-Scientific Research in the Public Interest (201503109).

\section{Conflict of interest}

The authors declare that the research was conducted in the absence of any commercial or financial relationships that could be construed as a potential conflict of interest.

\section{References}

1. Alstrom S (2001) Characteristics of bacteria from oilseed rape in relation of their biocontrol activity against Verticillium dahliae. J Phytopathol 149:57-64

2. Akao $S$ (2017) Nitrogen, phosphorus, and antioxidant contents in crop residues for potential cascade utilization. Waste Biomass Valori 4:1-8

3. Atallah ZK, Maruthachalam K, Subbarao KV (2012) Sources of Verticillium dahliae affecting lettuce. Phytopathology 102(11):1071-1078

4. Antoniou A, Tsolakidou MD, Stringlis IA et al (2017) Rhizosphere microbiome recruited from a suppressive compost improves plant fitness and increases protection against vascular wilt pathogens of tomato. Front Plant Sci 29(8): 2022 
5. Azabou MC, Gharbi Y, Medhioub I et al (2019) The endophytic strain Bacillus velezensis oee1: an efficient biocontrol agent against verticillium wilt of olive and a potential plant growth promoting bacteria. Biol Control 142:104168

6. Bastida F, Torres IF, ABbadia J et al (2018) Comparing the impacts of drip irrigation by freshwater and reclaimed wastewater on the soil microbial community of two citrus species. Agr Water Manage 203:53-62

7. Bulluck LR, Brosius M, Evanylo GK et al (2002) Organic and synthetic fertility amendments influence soil microbial, physical and chemical properties on organic and conventional farms. Appl Soil Ecol 19(2):147-160

8. Chamberlain LA, Bolton ML, Cox MS et al (2020) Crop rotation, but not cover crops, influenced soil bacterial community composition in a corn-soybean system in southern wisconsin. Appl Soil Ecol 154:103603

9. Cui J, Wang JJ, Xu J et al (2017) Changes in soil bacterial communities in an evergreen broad-leaved forest in east China following 4 years of nitrogen addition. J Soil Sediment 17(8):2156-2164

10. Davis JR, Huisman OC, Everson DO et al (2010) The suppression of verticillium wilt of potato using corn as a green manure crop. Am J Potato Res 87:195-208

11. Díaz-Pérez JC, Phatak SC, Ruberson J et al (2012) Mulches increase yield and improve weed control in no-till organic broccoli (brassica oleracea var. botrytis). Acta Hortic 933:337-342

12. Fradin EF, Thomma BP (2006) Physiology and molecular aspects of verticillium wilt diseases caused by $V$. dahliae and $V$. alboatrum. Mol Plant Pathol 7:71-86

13. Gamliel A, Austerweil M, Kritzman G (2000) No-chemical approach to soilborne pest management organic amendments. Crop Prot 19:847-853

14. Garbeva P, Van VJ, Van EJ (2004) Microbial diversity in soil: selection of microbial populations by plant and soil type and implications for disease suppressiveness. Annu Rev Phytopathol 42:243270

15. Gong XY, Shi JB, Zhou XG et al (2021) Crop rotation with cress increases cucumber yields by regulating the composition of the rhizosphere soil microbial community. Front Microbiol 12:556

16. Göre ME, Caner ÖK, Altın N et al (2009) Evaluation of cotton cultivars for resistance to pathotypes of Verticillium dahliae. Crop Prot 28(3):215-219

17. Guerra PC, Keil CB, Stevenson PC et al (2017) Larval performance and adult attraction of Delia platura (Diptera: Anthomyiidae) in a native and an introduced crop. J Econ Entomol 110(1):186-191

18. Hao LY, Zheng XL, Wang Y et al (2019) Inhibition of tomato early blight disease by culture extracts of a streptomyces puniceus isolate from mangrove soil. Phytopathology 109(7):1149-1156

19. Housam K, Yitzhak H, Shlomit M et al (2018) Effect of compost properties on progress rate of Verticillium dahliae attack on eggplant (Solanum melongena L.). Compost Sci UtiL 26(2):71-78

20. Inderbitzin P, Ward J, Barbella A et al (2018) Soil microbiomes associated with Verticillium wiltsuppressive broccoli and chitin amendments are enriched with potential biocontrol agents. 
Phytopathology 108:31-43

21. Ikeda K, Banno S, Furusawa A et al (2015) Crop rotation with broccoli suppresses Verticillium wilt of eggplant. J Gen Plant Pathol 81:1-6

22. Johnson DA, Dung JKS (2010) Verticillium wilt of potato-the pathogen, disease and management. Can J Plant Pathol 32(1):58-67

23. Kavino M, Harish S, Kumar N et al (2010) Effect of chitinolytic PGPR on growth, yield and physiological attributes of banana (Musa spp.) under field conditions. Appl Soil Ecol 45:71-77

24. Klosterman SJ, Atallah ZK, Vallad GE et al (2009) Diversity, pathogenicity, and management of verticillium species. Annu Rev Phytopathol 47:39-62

25. Koike ST, Subbarao KV (2000) Broccoli residues can control Verticilliurn wilt of cauliflower. Calif Agr 54(3):30-33

26. Li CH, Feng ZL, Li ZF et al (2015) In vitro sensitivity of Verticillium dahliae Kleb strains against some effective fungicides. China Cotton 42:16-18

27. Li SZ, Ma P, Gao SG et al (2005) Evaluation of biocontrol potential of a bacterial strain NCD-2 against cotton Verticillium wilt in field trials. Acta Phytopathologica Sinica 35:451-455

28. López-Pérez JA, Roubtsova T, Ploeg A (2005) Effect of three plant residues and chicken manure used as biofumigants at three temperatures on Meloidogyne incognita infestation of tomato in greenhouse experiments. J Nematol 37(4):489-494

29. Lu R, Li YP, Li WX et al (2018) Bacterial community structure in atmospheric particulate matters of different sizes during the haze days in xi'an, China. Sci Total Environ 637-638:244-252

30. Lugtenberg B, Kamilova F (2009) Plant-growth-promoting rhizobacteria. Annu Rev Microbiol 63:541556

31. Manuel A, Celia B (2017) Identifying characteristics of verticillium wilt suppressiveness in olive mill composts. Plant Dis 101(9):1568-1577

32. Pandin C, Védie R, Rousseau T et al (2018) Dynamics of compost microbiota during the cultivation of Agaricus bisporus in the presence of Bacillus velezensis QST713 as biocontrol agent against Trichoderma aggressivum. Biol Control 127:39-54

33. Poulsen PHB, Al-Soud WA, Bergmark L et al (2013) Effects of fertilization with urban and agricultural organic wastes in a field trial Prokaryotic diversity investigated by pyrosequencing. Soil Biol Biochem 57:784-793

34. Puopolo G, Palmieri MC, Giovannini O. Pertot I (2015) Impact of temperature on the survival and the biocontrol efficacy of Lysobacter capsiciaz 78 against Phytophthora infestans. Biocontrol 60(5):681-689

35. Qiu MH, Zhang RF, Xue C et al (2012) Application of bioorganic fertilizer can control Fusarium wilt of cucumber plants by regulating microbial community of rhizosphere soil. Biol Fert Soils 48:807-816

36. Rehman L, Su XF, Li XK et al (2018) FreB is involved in the ferric metabolism and multiple pathogenicity-related traits of Verticillium dahliae. Curr Genet 64(3):645-659 
37. Romanyà J, Sancho-Adamson M, Ortega D et al (2019) Early stage effects of verticillium wilt of olive (WVO) on nutrient use in young olive trees grown in soils amended with compost and mineral fertilisation. Plant Soil 436:193-209

38. Roy T, Bandopadhyay A, Sonawane PJ et al (2018) Bio-effective disease control and plant growth promotion in lentil by two pesticide degrading strains of Bacillus sp. Biol Control 127:55-63

39. Saravanan T, Muthusamy M, Marimuthu T (2003) Development of integrated approach to manage the fusarial wilt of banana. Crop Prot 22(9):1117-1123

40. Shen ZZ, Zhong ST, Wang YG et al (2013) Induced soil microbial suppression of banana fusarium wilt disease using compost and biofertilizers to improve yield and quality. Eur J Soil Biol 57:1-8

41. Shen ZZ, Wang DS, Ruan YZ et al (2014) Deep 16S rRNA pyrosequencing reveals a bacterial community associated with banana Fusarium wilt disease suppression induced by bio-organic fertilizer application. PLoS ONE 9(5):e98420

42. Stringlis IA, Yu K, Feussner K et al (2018) MYB72-dependent coumarin exudation shapes root microbiome assembly to promote plant health. Proc Natl Acad Sci 115:5213-5222

43. Subbarao KV, Hubbard JC, Koike ST (1999) Evaluation of broccoli residue incorporation into field soil for verticillium wilt control in cauliflower. Plant Dis 83:124-129

44. Tao KY, Zhang XY, Chen XP et al (2019) Response of soil bacterial community to bioaugmentation with a plant residue-immobilized bacterial consortium for crude oil removal. Chemosphere 222:831838

45. Taylor RJ, Pasche JS, Gudmestad NC (2005) Influence of tillage and method of metam sodium application on distribution and survival of Verticillium dahliae, in the soil and the development of Verticillium wilt of potato. Am J Potato Res 82(6):451-461

46. Tian $\mathrm{H}$, Wang $\mathrm{H}$, Hui $\mathrm{XL}$ et al (2017) Changes in soil microbial communities after 10 years of winter wheat cultivation versus fallow in an organic-poor soil in the Loess Plateau of China. PLoS ONE 12(9):e0184223

47. Tubeileh AM, Stephenson GT (2020) Soil amendment by composted plant wastes reduces the Verticillium dahliae abundance and changes soil chemical properties in a bell pepper cropping system. Curr Plant Biol 22:100148

48. Uppal AK, El HA, Adams LR et al (2008) Biological control of potato verticillium wilt under controlled and field conditions using selected bacterial antagonists and plant extracts. Biol Control 44(1):90100

49. Wang ML, Geng LL, Sun XX et al (2020) Screening of Bacillus thuringiensis strains to identify new potential biocontrol agents against Sclerotinia sclerotiorum and Plutella xylostella in Brassica campestris L. Biol Control 145: 104262

50. Wang YJ, Zhao WS, Chen D et al (2020) Effect of growth substrate amended with broccoli residues on the spread of GFP-labeled Verticillium dahliae strain in cotton plant. Acta Phytopathologica Sinica 50(1):89-96 
51. Wheeler DL, Johnson DA (2016) Verticillium dahliae infects, alters plant biomass, and produces inoculum on rotation crops. Phytopathology 106(6):602-613

52. Wiggins BE, Kinkel LL (2005) Green manures and crop sequences influence potato diseases and pathogen inhibitory activity of indigenous streptomycetes. Phytopathology 95:178-185

53. Yin CT, Hulbert SH, Schroeder KL et al (2013) Role of bacterial communities in the natural suppression of Rhizoctonia solani bare patch of wheat (Triticum aestivum L.). Appl Environ Microb 79(23):7428-7438

54. Zhang JY, Liu YX, Zhang N et al (2019) NRT1.1B is associated with root microbiota composition and nitrogen use in field-grown rice. Nat Biotechnol 37:676-684

55. Zhang YL, Li ZF, Feng ZL et al (2016) Functional analysis of the pathogenicity-related gene VdPR1 in the vascular wilt fungus Verticillium dahliae. PLoS ONE 11(11):e0166000

56. Zhao WS, Guo QG, Li SZ et al (2021) Effects of Bacillus subtilis NCD-2 and broccoli residues return on potato verticillium wilt and soil fungal community structure. Biol Control 159:104628

57. Zhao WS, Guo QG, Li SZ et al (2019a) Control efficacy of broccoli residues on cotton verticillium wilt and its effect on soil bacterial community at different growth stages. Scientia Agricultura Sinica 52(24):4505-4517

58. Zhao WS, Guo QG, Li SZ et al (2020) Effect of wilt-resistant and wilt-susceptible cotton on soil bacterial community structure at flowering and boll stage. Scientia Agricultura Sinica 53(5):942-954

59. Zhao WS, Li SZ, Lu XY et al (2019b) Safety evaluation of broccoli residues returning on cotton growth and its control effect on verticillium wilt. Chinese Journal of Biological Control 35(3):449455

60. Zhao WS, Guo QG, Zhang XY et al (2020) Development of microbial agent Bacillus amyloliquefaciens PHODG36 and its effect on disease control and yield increase of potato. Chinese Journal of Biological Control 36(3):381-387

61. Zhou J, Guan DW, Zhou BK et al (2015) Influence of 34-years of fertilization on bacterial communities in an intensively cultivated black soil in Northeast China. Soil Biol Bioch 90:42-51

62. Zhou WP, Shen WJ, Li YE et al (2017) Interactive effects of temperature and moisture on composition of the soil microbial community. Eur J Soil Sci 68(6):909-918

\section{Figures}



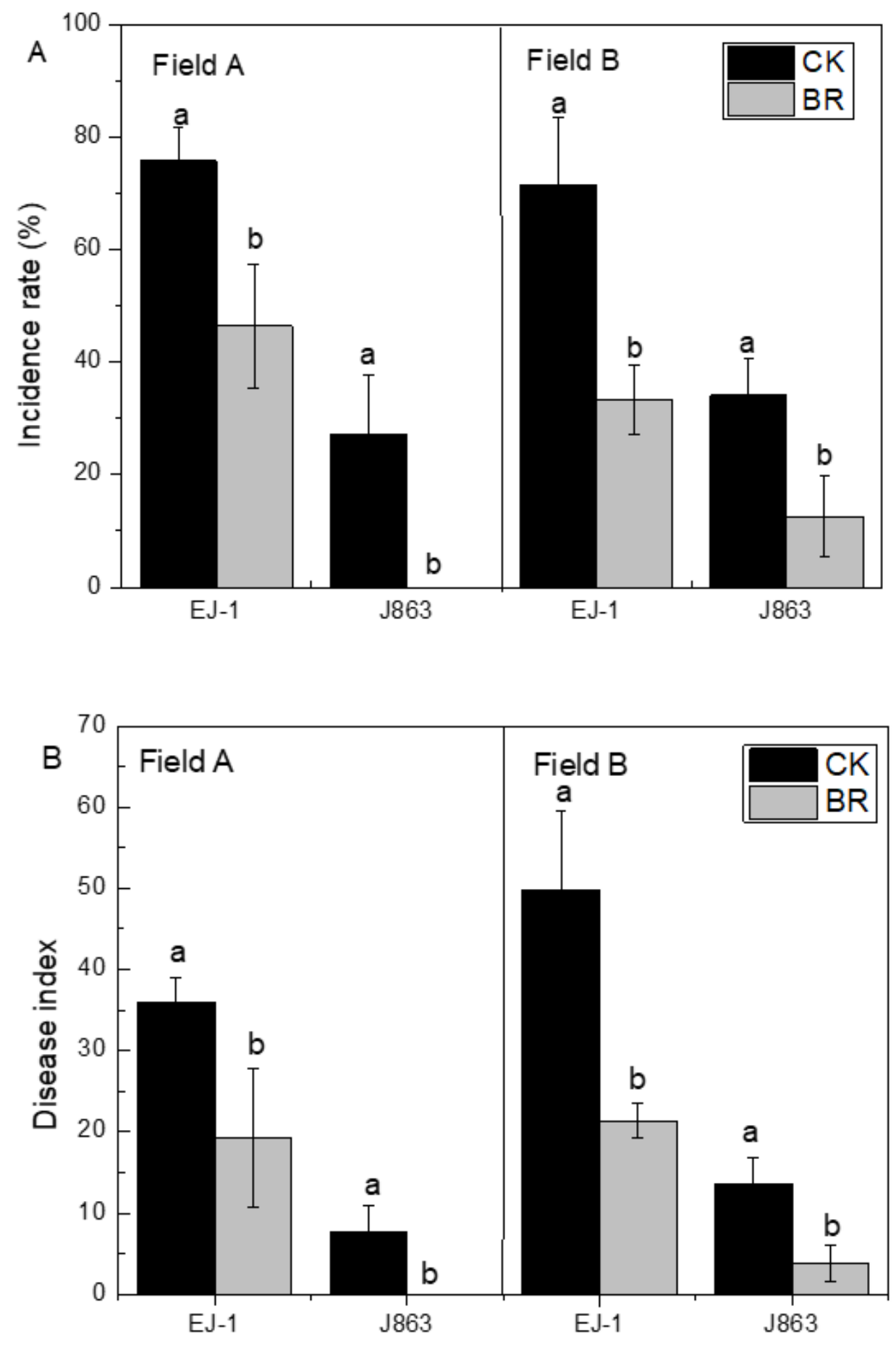

Figure 1

Comparison of the occurrence of verticillium wilt of different cotton cultivars following the application of broccoli residues 


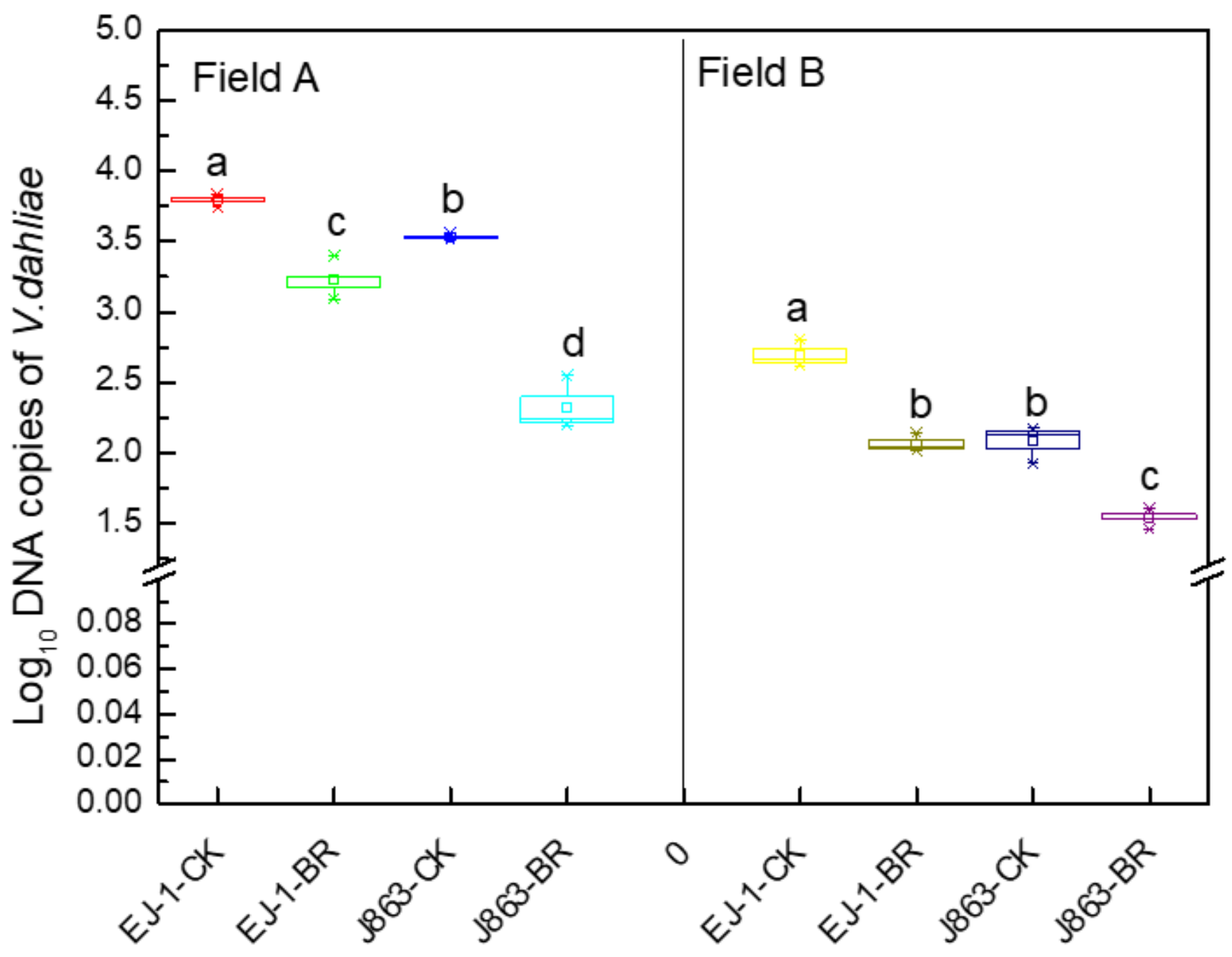

Figure 2

Quantification of V. dahliae in soil by real-time PCR 

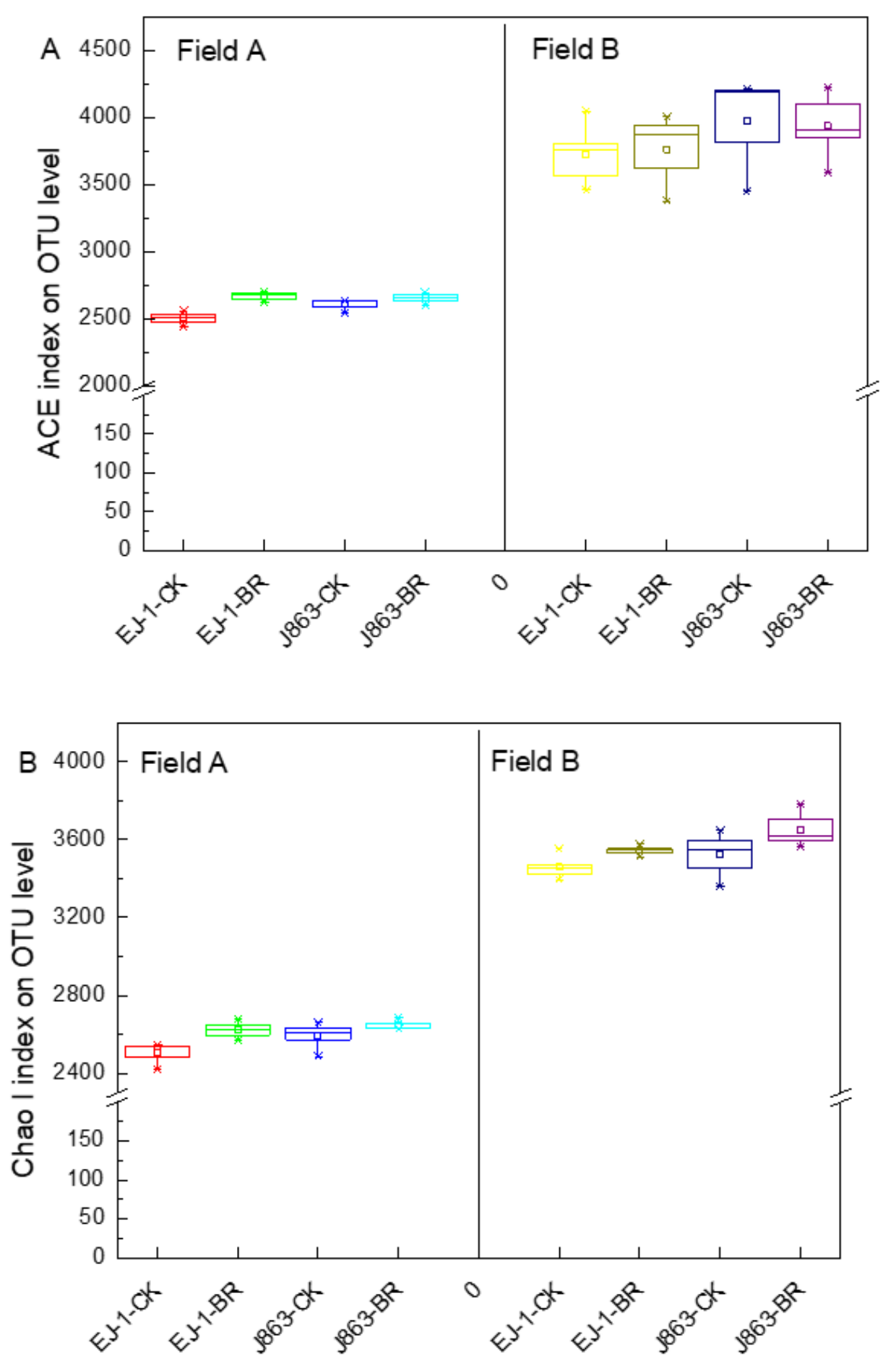

Figure 3

Effect of the BR treatment on the alpha diversity indices of the bacterial community associated with different cotton cultivars 

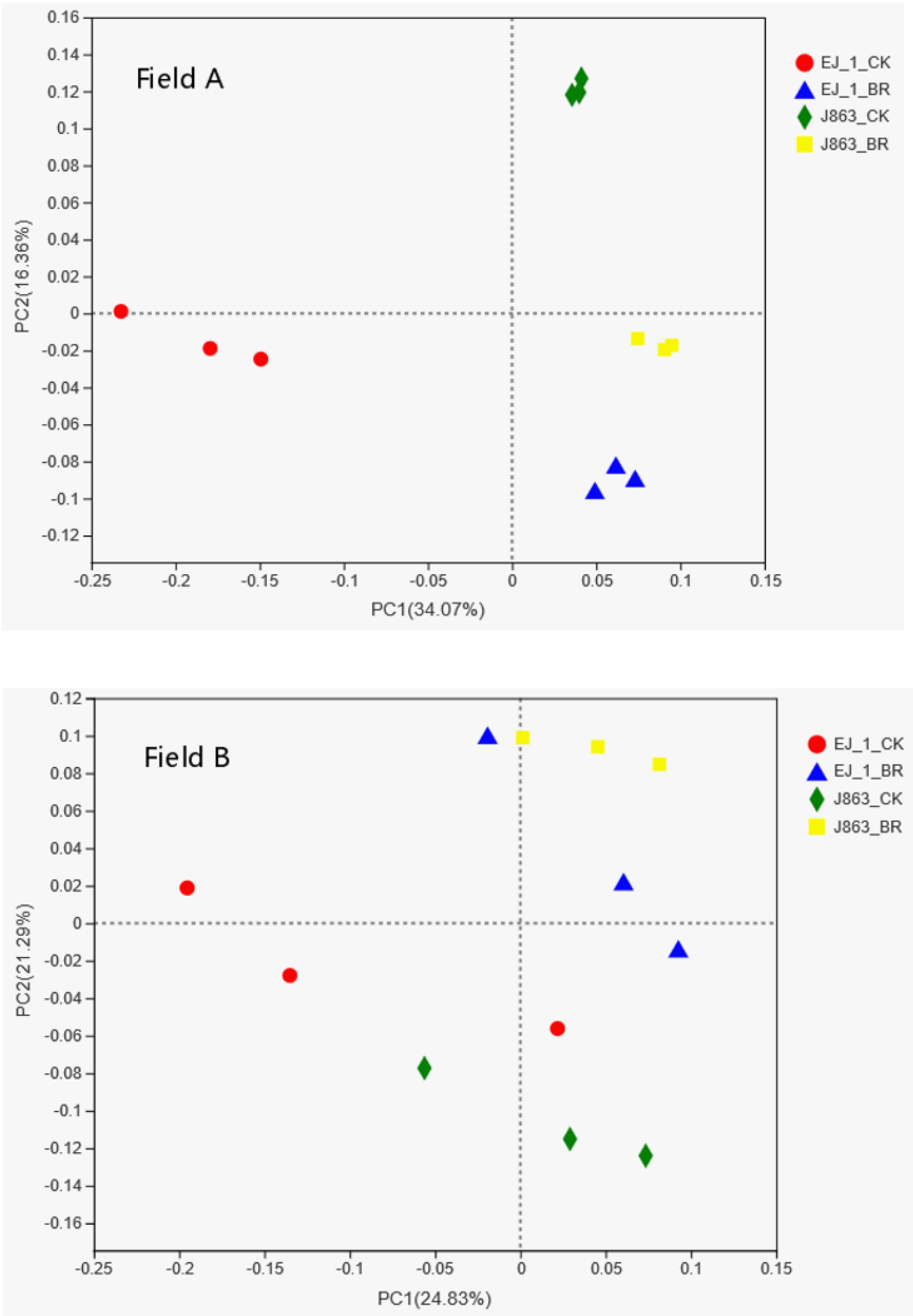

Figure 4

PCA of the soil bacterial community associated with different cotton cultivars at the OTU level following the application of broccoli residues 

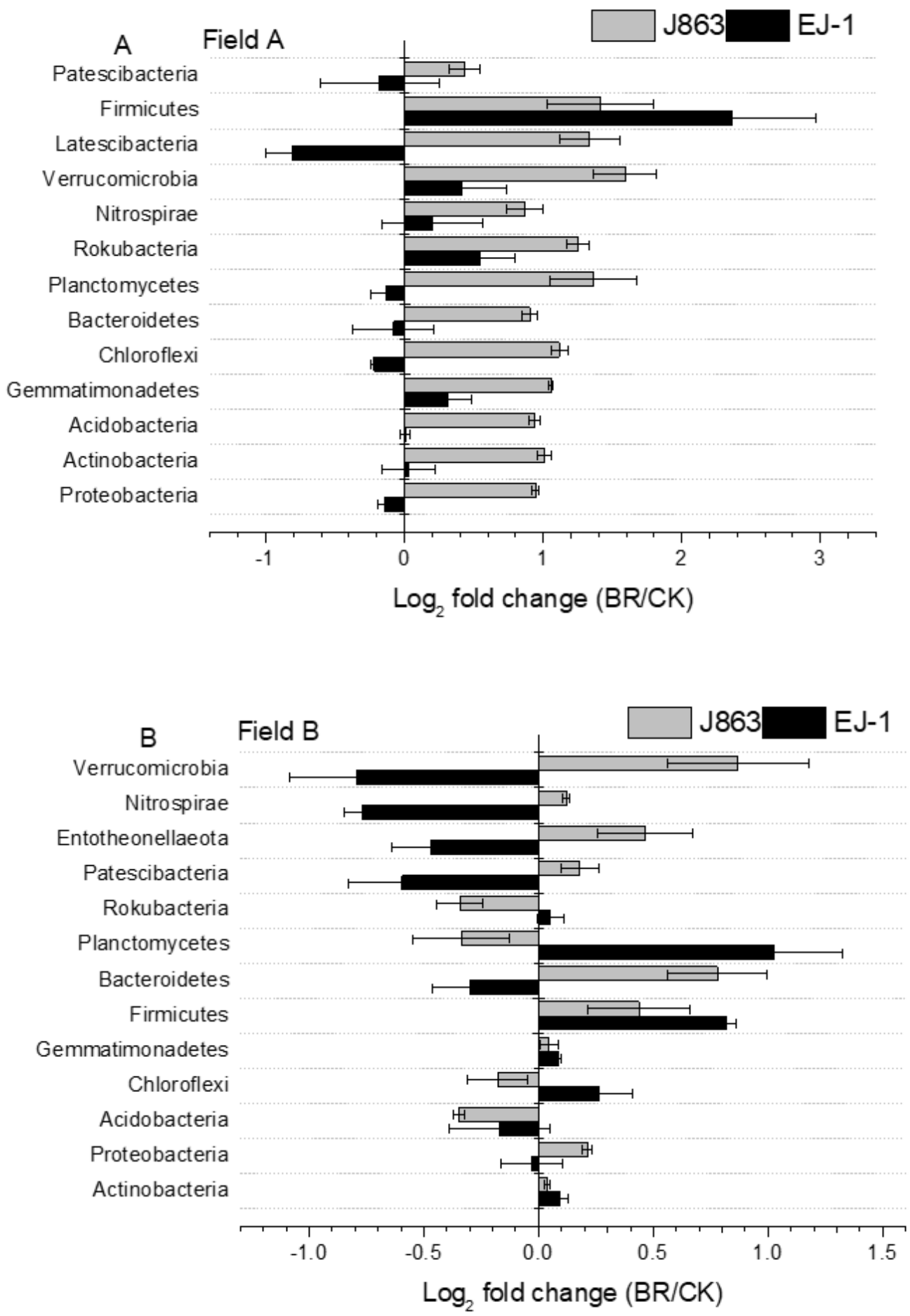

\section{Figure 5}

Comparison of the dominant bacterial taxa associated with the different cotton varieties after the application of broccoli residues at the phylum level 

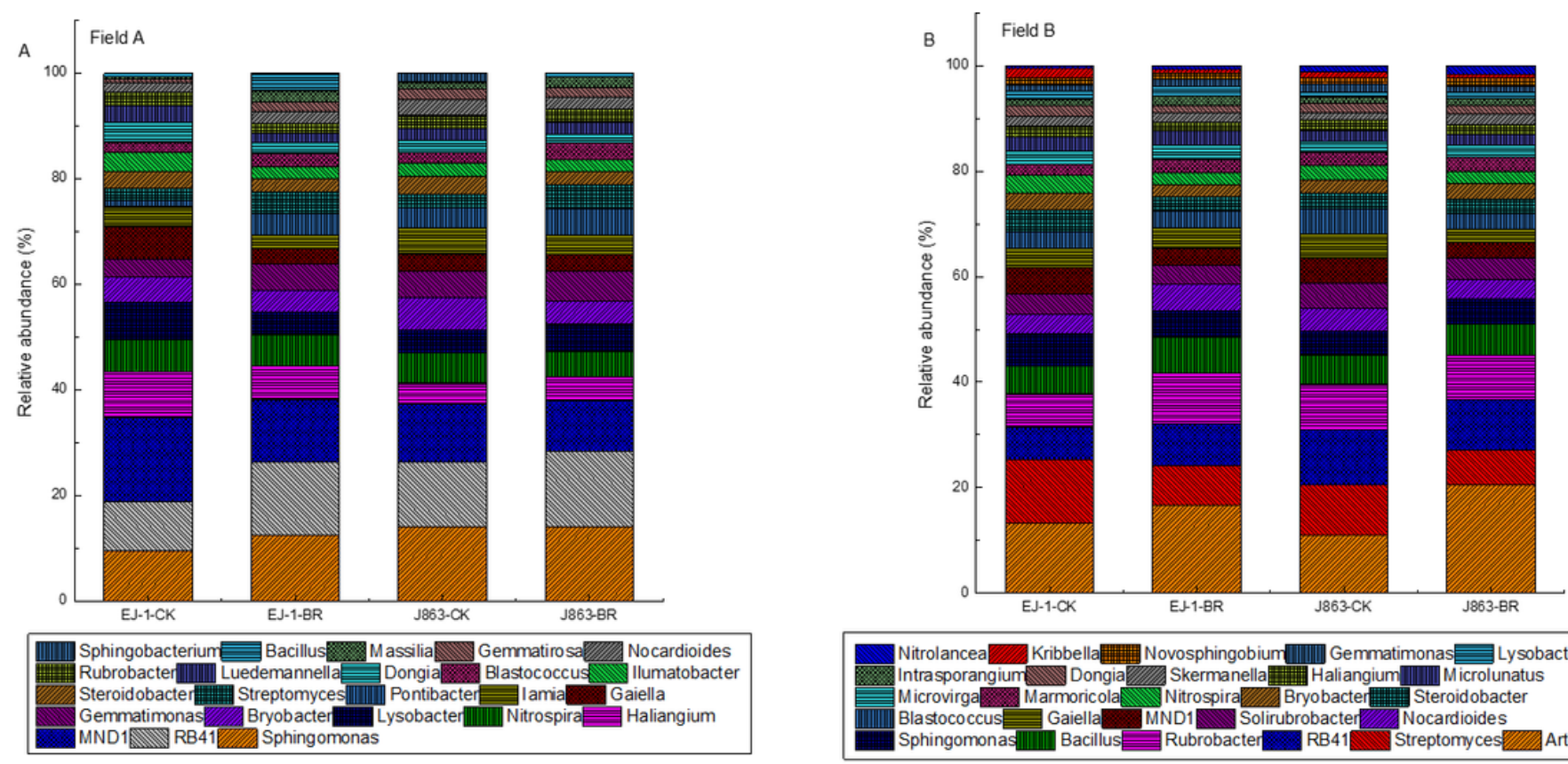

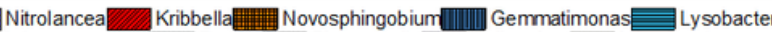
Intrasporangium Dongia Skermanella Haliangium Mind Microlunatus Microvirga Marmoricola Nitrospiram Bryobacter Blastococcus Gaiella MND1 Solirubrobacter Nocardioides

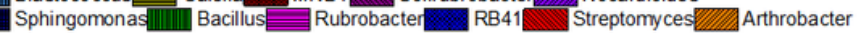

Figure 6

Changes in the relative abundances of the bacterial taxa associated with different cotton cultivars at the genus level following the application of broccoli residues 

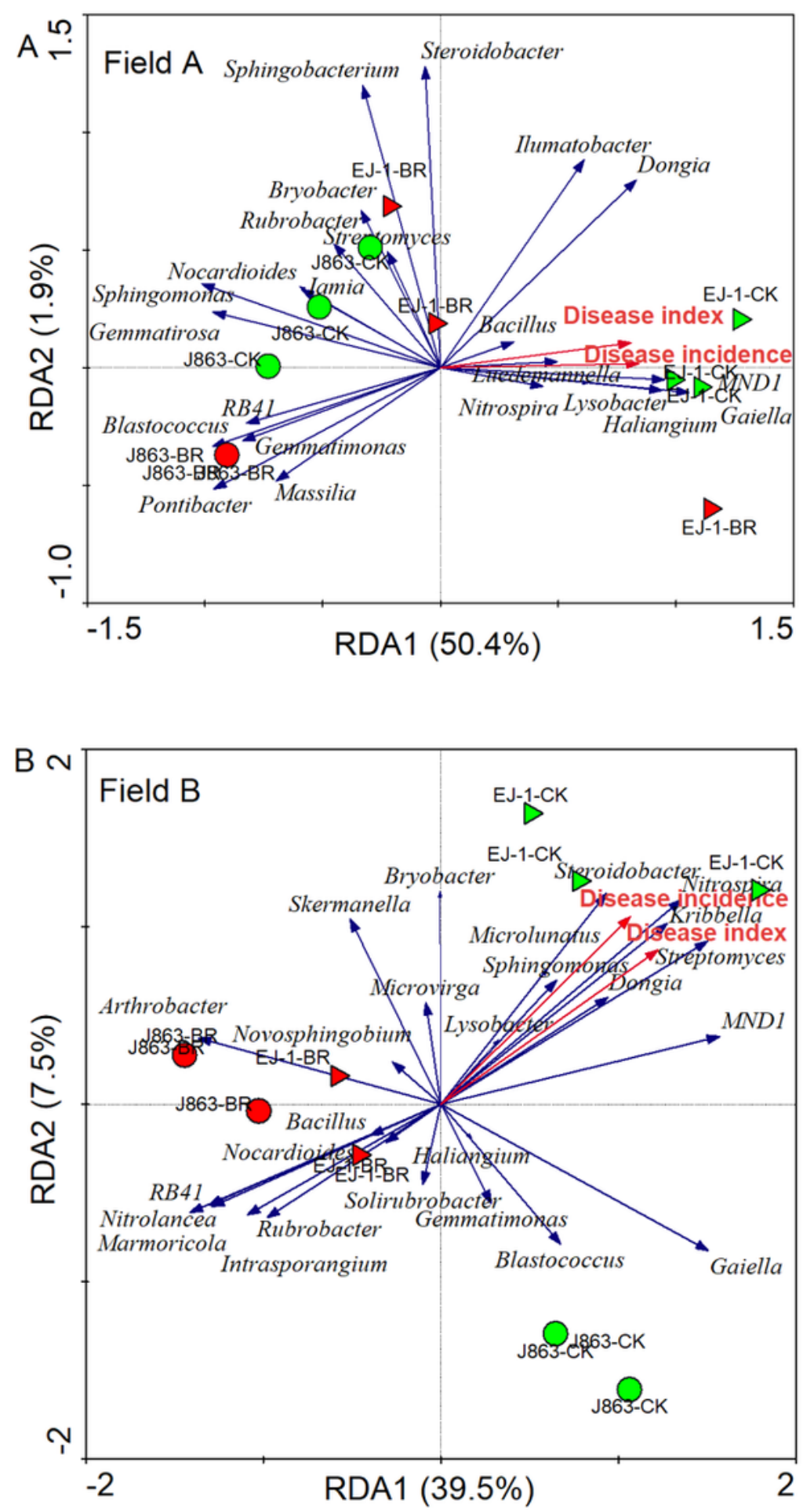

\section{Figure 7}

Redundancy analysis (RDA) of the relative abundance of genera and verticillium wilt disease occurrence for different cotton cultivars and different treatments 


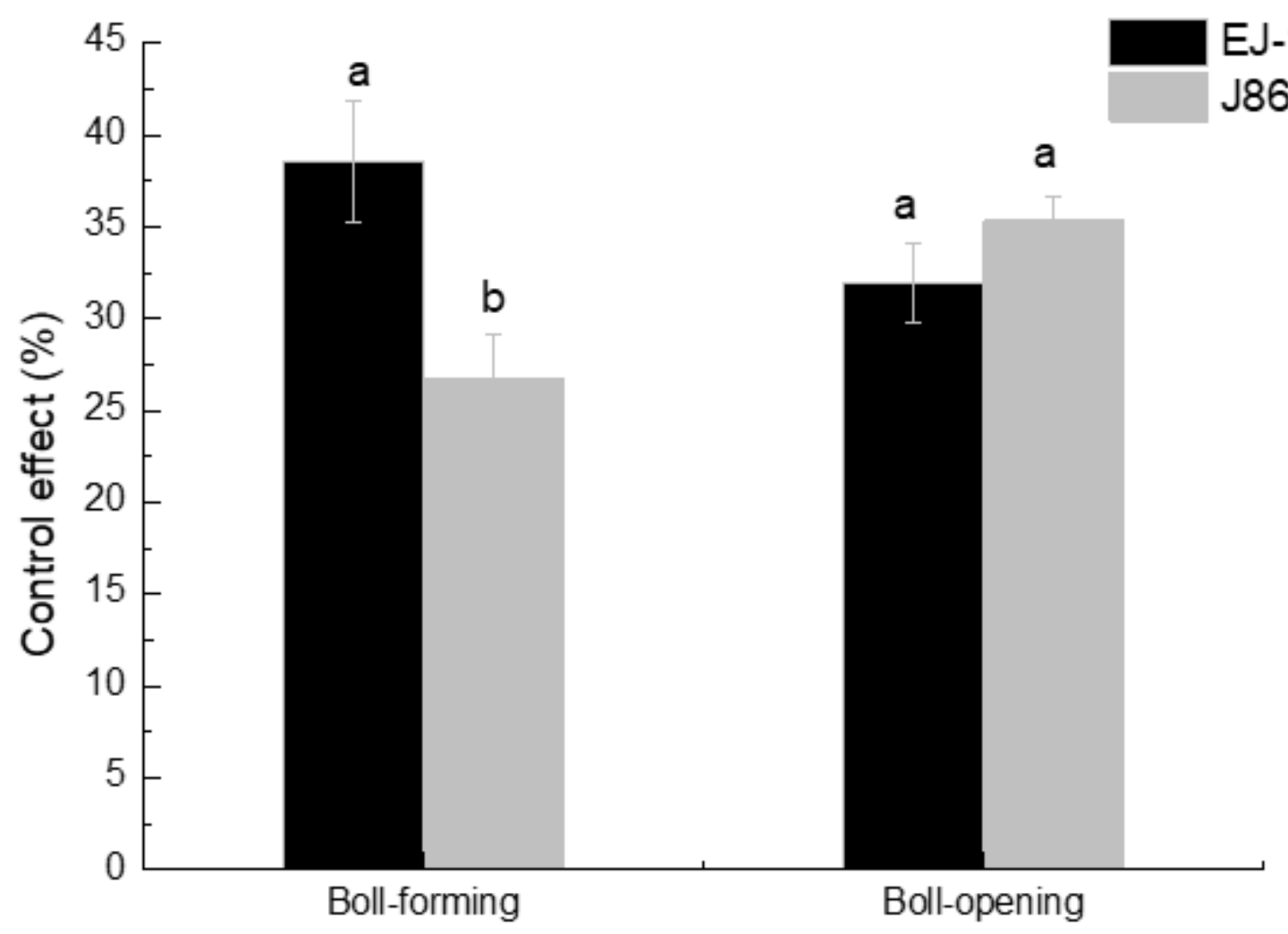

Figure 8

Control effect of Bacillus subtilis NCD-2 on verticillium wilt of cotton at different stages

\section{Supplementary Files}

This is a list of supplementary files associated with this preprint. Click to download.

- FigS1.png

- Figs2.png 